\title{
Developing and validating predictive decision tree models from mining chemical structural fingerprints and high-throughput screening data in PubChem
}

\author{
Lianyi Han, Yanli Wang* and Stephen H Bryant*
}

Address: National Center for Biotechnology Information, National Library of Medicine, National Institutes of Health, Bethesda, MD 20894, USA

Email: Lianyi Han - hanl@ncbi.nlm.nih.gov; Yanli Wang* - ywang@ncbi.nlm.nih.gov; Stephen H Bryant* - bryant@ncbi.nlm.nih.gov

* Corresponding authors

Published: 25 September 2008

BMC Bioinformatics 2008, 9:40I doi:10.1186/1471-2105-9-40I

This article is available from: http://www.biomedcentral.com/l47|-2/05/9/40।

(c) 2008 Han et al; licensee BioMed Central Ltd.

This is an Open Access article distributed under the terms of the Creative Commons Attribution License (http://creativecommons.org/licenses/by/2.0), which permits unrestricted use, distribution, and reproduction in any medium, provided the original work is properly cited.

\begin{abstract}
Background: Recent advances in high-throughput screening (HTS) techniques and readily available compound libraries generated using combinatorial chemistry or derived from natural products enable the testing of millions of compounds in a matter of days. Due to the amount of information produced by HTS assays, it is a very challenging task to mine the HTS data for potential interest in drug development research. Computational approaches for the analysis of HTS results face great challenges due to the large quantity of information and significant amounts of erroneous data produced.
\end{abstract}

Results: In this study, Decision Trees (DT) based models were developed to discriminate compound bioactivities by using their chemical structure fingerprints provided in the PubChem system http://pubchem.ncbi.nlm.nih.gov. The DT models were examined for filtering biological activity data contained in four assays deposited in the PubChem Bioassay Database including assays tested for $5 \mathrm{HTla}$ agonists, antagonists, and HIV-I RT-RNase H inhibitors. The I0-fold Cross Validation (CV) sensitivity, specificity and Matthews Correlation Coefficient (MCC) for the models are $57.2 \sim 80.5 \%, 97.3 \sim 99.0 \%, 0.4 \sim 0.5$ respectively. A further evaluation was also performed for DT models built for two independent bioassays, where inhibitors for the same HIV RNase target were screened using different compound libraries, this experiment yields enrichment factor of 4.4 and 9.7.

Conclusion: Our results suggest that the designed DT models can be used as a virtual screening technique as well as a complement to traditional approaches for hits selection.

\section{Background}

High-throughput screening (HTS) is an automated technique and has been effectively used for rapidly testing the activity of large numbers of compounds [1-3]. Advanced technologies and availability of large-scale chemical libraries allow for the examination of hundreds of thousands of compounds in a day via HTS. Although the exten- sive libraries containing several million compounds can be screened in a matter of days, only a small fraction of compounds can be selected for confirmatory screenings. Further examination of verified hits from the secondary dose-response assay can be eventually winnowed to a few to proceed to the medicinal chemistry phase for lead optimization $[4,5]$. The very low success rate from the hits-to- 
lead development presents a great challenge in the earlier screening phase to select promising hits from the HTS assay [4]. Thus, the study of HTS assay data and the development of a systematic knowledge-driven model is in demand and useful to facilitate the understanding of the relationship between a chemical structure and its biological activities.

In the past, HTS data has been analyzed by various cheminformatics methods [6-17], such as cluster analysis[10], selection of structural homologs[11,12], data partitioning [13-16] etc. However, most of the available methods for HTS data analysis are designed for the study of a small, relatively diverse set of compounds in order to derive a Quantitative Structure Activity Relationship(QSAR) [1821] model, which gives direction on how the original collection of compounds could be expanded for the subsequent screening. This "smart screening" works in an iterated way for hits selection, especially for selecting compounds with a specific structural scaffold [22]. With the advances in HTS screening, activity data for hundreds of thousands' compound can be obtained in a single assay. Altogether, the huge amount of information and significant erroneous data produced by HTS screening bring a great challenge to computational analysis of such biological activity information. The capability and efficiency of analysis of this large volume of information might hinder many approaches that were primarily designed for analysis of sequential screening. Thus, in dealing with large amounts of chemicals and their bioactivity information, it remains an open problem to interpret the drug-target interaction mechanism and to help the rapid and efficient discovery of drug leads, which is one of the central topics in computer-aided drug design [23-30].

Although the (Quantitative) Structure Activity Relationship-(Q)SAR has been successfully applied in the regression analysis of leads and their activities [18-21], it is generally used in the analysis of HTS results for compounds with certain structural commonalities. However, when dealing with hundreds of thousands of compounds in a HTS screening, the constitution of SAR equations can be both complicated and impractical to describe explicitly.

Molecular docking is another widely used approach to study the relationship between targets and their inhibitors by simulating the interactions and binding activities of receptor-ligand systems or developing a relationship among their structural profiles and activities[31,32]. However, as it takes the interactions between the compounds and the target into consideration, it has been widely used for virtual screening other than to extract knowledge from experimental activities.
Decision Tree (DT) is a popular machine learning algorithm for data mining and pattern recognition. Compared with many other machine learning approaches, such as neural networks, support vector machines and instance centric methods etc., DT is simple and produces readable and interpretable rules that provide insight into problematic domains. DT has been demonstrated to be useful for common medical clinical problems where uncertainties are unlikely [33-37]. It has been applied to some bioinformatics and cheminformatics problems, such as characterizations of Leiomyomatous tumour[38], prediction of drug response[39], classification of antagonist of dopamine and serotonin receptors[40], virtual screening of natural products[41].

In this study, we propose a DT based model to generalize feature commonalities from active compounds tested in HTS screening. We utilized DT as the basis to develop the model because it has been successfully applied in many biological problems, and it is able to generate a set of rules from the active compounds which can then be used for filtering the untested compounds that are likely to be active in the biological system of interest. Moreover, it has the capability to handle the arbitrary degree of non-linear structurally diversified compounds.

Many elegant algorithms for building decision tree models have been introduced and applied in real life problems, and C4.5[42] is one of the best known programs for constructing decision trees. In this work, the DT based model was developed on the basis of the Decision Tree C4.5 algorithm[42]. The representation of the molecular structures is described by the PubChem fingerprint system. The DT based model was further examined by four assays deposited in the PubChem Bioassay Database, the HTS assay for 5-Hydroxytryptamine Receptor Subtype 1a (5HT1a) antagonists(PubChem AID:612), HTS assay for 5HT1a agonists(PubChem AID 567), and two other assays with PubChem AID 565 and 372 for screening the HIV-1 RT-RNase H inhibitors. The results of 10-fold Cross Validation (CV) over these HTS assays suggest the selfconsistency of the DT models. Since a model simply provides the rules based on the profiles of active compounds in a specific HTS assay, the computationally generated models were further examined using two HTS assays which tested the same HIV RNase target, but used different compound libraries and were performed independently by two individual research laboratories. Our results suggest that these developed models could be used to validate HTS assay data for noise reduction and to identify hits through virtual screening of additional and larger compound libraries. 


\section{Results and discussion Development of DT model}

In this study, four DT models were developed for activity data contained in PubChem bioassay AID 612, 567, 565 and 372 respectively, where compounds were screened for various activities against several protein targets (Table 1).

Model fitting accuracies are used to examine whether the proposed data model can handle the complex data and whether the chemical fingerprint descriptors are sufficient for model development. As shown in Table 2, the model fitting accuracies of the four DT models were in the range of $98.6 \%$ to $99.8 \%$. This suggested that the DT based models are able to fit the majority of the HTS data for the model generalization. Sensitivity and specificity of each developed model, which indicate the ratio of the active and inactive compounds that can be successfully learned by the DT model, are also reported in Table 2 . The sensitivities of the models built for AID 612, AID 567, AID 565 and AID 372 are $86.5 \%, 98.9 \%, 90.2 \%$ and $83.1 \%$ respectively, while the specificities are all greater than $98 \%$. The model fitting accuracies for both active and inactive compounds suggest strong feature-activity relationship among compounds tested in the HTS screenings. The small fraction of misrecognized compounds might result from the HTS data noise, the discrepancy of bioactivities observed from the compounds with same or similar chemical structures, or the competition with the overwhelming inactive compounds

As shown by the comparison of the sensitivity and its corresponding specificity for each individual DT model, the sensitivity is usually lower than the specificity and contributes less to the overall accuracy. One possible explanation is the existence of the data imbalance issue. Among the HTS data analyzed in this study, the ratio between the number of active compounds and that of inactives are ranged from 1:51 1:176. Thus comparing to the active compounds, the rules could be easily generalized for inactive compounds when the chance of pattern reoccurrence is higher. Data Imbalance problem is common in the high throughput screening assay data. One HTS assay could have tens of thousands of compounds tested and only yield few dozens of hits. Due to this problem, the specificity becomes less objective in performance evaluation. Therefore, we also use the Matthews Correlation Coefficient (MCC) [43] as additional measure to evaluate the model's performance.

MCC took both sensitivity and specificity into account and it is generally used as a balanced measure in dealing with data imbalance situation. As shown in Table 2, MCC values fall in the range of $0.67 \sim 0.84$ for the four HTS assays, which again suggests the satisfactory performance of the model training and indicates that the self recognition of the model is not random.

\section{Model validation by self-consistency test}

The validation of the DT based models and self-consistency test were performed by 10 -fold cross validation (CV) method, in which the compound dataset tested in one HTS assay was randomly split into 10 folds. These models were set up using 9 randomly selected folds, and prediction was done on the remaining fold.

The 10-fold CV results are given in Table 3. The overall validation accuracies of all DT models ranged from $96.9 \%$ to $98.9 \%$. While the sensitivities of the models built for AID 612, AID 567, AID 565 and AID 372 are 64.5\%, $80.5 \%, 75.2 \%$ and $57.2 \%$ respectively, where the specificities were $99.1 \%, 99.0 \%, 97.3 \%$ and $98.9 \%$ respectively. The more than $96 \%$ overall accuracies of the four DT based models suggest overall good performance and the CV analysis validates the reliability of the DT based models.

The sensitivity and specificity values given here represent the classification accuracies for the active and inactive compounds respectively. The sensitivity is lower than specificity to a certain extent. For example, the DT model for the 4HTa antagonist activity data demonstrates $64.5 \%$ sensitivity but $99.1 \%$ specificity. From the evidence given in the previous section, imbalanced data, data noise and data discrepancy could again account for the lower sensitivities. Moreover, as about $90 \%$ percent of the data used for training during the cross validations, the generaliza-

Table I: HTS assays analyzed in this study

\begin{tabular}{lllll}
\hline Protein Target & $\begin{array}{l}\text { The role of active } \\
\text { compounds }\end{array}$ & $\begin{array}{l}\text { PubChem Bioassay AID } \\
\text { No. }\end{array}$ & $\begin{array}{l}\text { Number of Compounds } \\
\text { tested }\end{array}$ & $\begin{array}{l}\text { Number of active } \\
\text { compounds identified }\end{array}$ \\
\hline $\begin{array}{l}\text { 5-Hydroxytryptamine } \\
\begin{array}{l}\text { Receptor Subtype Ia } \\
\text { HIV-I reverse }\end{array}\end{array}$ & agonist & 567 & 64,906 & 366 \\
$\begin{array}{l}\text { antagonist } \\
\text { rranscriptase associated } \\
\text { ribonuclease H }\end{array}$ & inhibitor & 612 & 61,606 & 416 \\
& 565 & 65,216 & 1,250 & 770 \\
\hline
\end{tabular}


Table 2: Recognition rate of Decision Tree models

\begin{tabular}{|c|c|c|c|c|c|c|c|c|c|c|}
\hline \multirow[b]{2}{*}{ Bioassay } & \multirow[b]{2}{*}{$\begin{array}{l}\text { PubChem } \\
\text { Assay ID }\end{array}$} & \multicolumn{3}{|c|}{ Active compounds } & \multicolumn{3}{|c|}{ Inactive compounds } & \multirow[b]{2}{*}{$\begin{array}{l}\text { Overall } \\
\text { accuracy }\end{array}$} & \multirow[b]{2}{*}{ MCC } & \multirow[b]{2}{*}{$\begin{array}{l}\text { Model } \\
\text { Complexity } \\
\text { (Number of } \\
\text { Nodes/ } \\
\text { Number of } \\
\text { Leaves/ } \\
\text { Number of } \\
\text { Features) }\end{array}$} \\
\hline & & TP & $\mathbf{F N}$ & Sensitivity & $\mathbf{T N}$ & $\mathbf{F P}$ & Specificity & & & \\
\hline $5 \mathrm{HTI}$ a agonist & 567 & 362 & 4 & $98.9 \%$ & 64,394 & 146 & $99.8 \%$ & $99.8 \%$ & 0.84 & $(321 / 161 / 149)$ \\
\hline $\begin{array}{l}5 \mathrm{HTIa} \\
\text { antagonist }\end{array}$ & 612 & 360 & 56 & $86.5 \%$ & 60,909 & 281 & $99.5 \%$ & $99.5 \%$ & 0.70 & $(1135 / 568 / 26 \mid)$ \\
\hline $\begin{array}{l}\text { HIV-I RT RNase } \\
\mathrm{H} \text { inhibitor }\end{array}$ & 565 & 1,128 & 122 & $90.2 \%$ & 63,070 & 896 & $98.6 \%$ & $98.4 \%$ & 0.70 & $(3003 / I 502 / 4 \mid 2)$ \\
\hline $\begin{array}{l}\text { HIV-I RT RNase } \\
\mathrm{H} \text { inhibitor }\end{array}$ & 372 & 640 & 130 & $83.1 \%$ & 98,463 & 535 & $99.5 \%$ & $99.3 \%$ & 0.67 & $(2511 / / 256 / 370)$ \\
\hline
\end{tabular}

TP = true positives, the number of correctly recognized active compounds;

$\mathrm{FN}=$ false negative, the number of active compounds that the model is unable to recognize;

$\mathrm{TN}=$ true negative, the number of inactive compounds that successfully recognized by the model:

$\mathrm{FP}=$ false positive, the number of inactive compounds that the model is unable to recognize.

Table 3: Performance evaluation of Decision Tree models by 10 fold Cross Validation.

\begin{tabular}{|c|c|c|c|c|c|c|c|c|c|}
\hline \multirow[b]{2}{*}{ Bioassay } & \multirow[b]{2}{*}{ PubChem Assay ID } & \multicolumn{3}{|c|}{ Active compounds } & \multicolumn{3}{|c|}{ Inactive compounds } & \multirow[b]{2}{*}{ Overall accuracy } & \multirow[b]{2}{*}{ MCC } \\
\hline & & TP & $\mathbf{F N}$ & Sensitivity & TN & $\mathbf{F P}$ & Specificity & & \\
\hline 5HTla agonist & 567 & 295 & 71 & $80.5 \%$ & 63913 & 627 & $99.0 \%$ & $98.9 \%$ & 0.50 \\
\hline $5 \mathrm{HTI}$ a antagonist & 612 & 268 & 148 & $64.5 \%$ & 60656 & 534 & $99.1 \%$ & $98.9 \%$ & 0.46 \\
\hline $\begin{array}{l}\text { HIV-I RT RNase H } \\
\text { inhibitor }\end{array}$ & 565 & 940 & 310 & $75.2 \%$ & 62269 & 1698 & $97.3 \%$ & $96.9 \%$ & 0.50 \\
\hline $\begin{array}{l}\text { HIV-I RT RNase H } \\
\text { inhibitor }\end{array}$ & 372 & 441 & 329 & $57.2 \%$ & 97923 & 1075 & $98.9 \%$ & $98.6 \%$ & 0.40 \\
\hline
\end{tabular}

TP = true positives, the number of correctly recognized active compounds;

$\mathrm{FN}=$ false negative, the number of active compounds that the model is unable to recognize;

$\mathrm{TN}=$ true negative, the number of inactive compounds that successfully recognized by the model;

$\mathrm{FP}=$ false positive, the number of inactive compounds that the model is unable to recognize. 
tion ability of the active compound dataset became easily affected due to the limited sample size as compared to that of the inactive compound dataset.

The learning capability of the DT model could also be affected by the way the model was trained, such as the minimum count of compound instances required for a decision node. However, it primarily depends on the datasets used for training. Although the imbalanced active and inactive compound datasets have an effect on the performance of the 10-fold CV, our results still show that the models are self-consistent. In addition, compounds and their activity data in HTS screens are able to converge toward a discrimination model with encouraging accuracies. In addition, the MCC values ranged from 0.4 to 0.5 , again indicating the potential of the models to identify potential hits.

\section{Application of DT models to select potential active compounds}

In this study, independent evaluation of the DT based models was attempted by using two HTS assays, PubChem AID 565 and 372, which were aimed at identifying HIV-1 RT RNase H inhibitors.

Comparison of the compound libraries of these two HTS assays were first performed, demonstrating the extent of similarity of the active compounds between the two assays. By using Tanimoto coefficient $[11,12]$ as a measurement for the compound similarities, there are only six active compounds that were found to be similar with Tanimoto coefficient threshold of $95 \%$. This suggested the overlap of the active compounds in these two assays was very limited. It maybe of interest to investigate whether the DT model built with one compound set can be used to filter out hits identified with another assay where a different compound library was screened. To this end, DT models of these two HTS assays were first developed independently and then each model was applied to classify the compounds screened by the other assay. An enrichment factor, which simply describes the proportion of active compounds from any given collection compared with randomly picked compounds [44], was calculated as assessment for the classification performance of each model.

Assume N compounds are tested in a HTS assay sample where A compounds have been experimentally verified as bioactive. By virtual screening, which is the activity classification using DT model in this study, $\mathrm{N}_{\mathrm{s}}$ compounds are predicted as active and among these $\mathrm{N}_{\mathrm{a}}$ belongs to the group of known bioactive compounds. A randomly picked sample will on average contain $\mathrm{AN}_{\mathrm{s}} / \mathrm{N}$ active molecules. Therefore, the formula for calculating the enrichment factor is $\mathrm{N}_{\mathrm{a}} \mathrm{N} / \mathrm{N}_{\mathrm{s}} \mathrm{A}$.
The enrichment factors for cross dataset prediction of HIV RNase H inhibitors of AID 372 and 565 are 4.4 and 9.7 respectively. From the virtual screening point of view, which is focusing on selecting the true hits while excluding the false positives as much as possible, the results suggest that the model derived from these two bioassays have certain generalization abilities to increase the odds of selecting true hits.

On the other hand, the sensitivities of the DT models based on data sets AID 372 and 565 are $0.4 \%$ and $6.9 \%$, and the specificities are $98.5 \%$ and $99.9 \%$ respectively, which yield corresponding MCC value of 0.03 and 0.04 , apparently the sensitivities and MCC values in this experiment are "poor" comparing to the cross validation study. This is not surprising, and indeed is well expected as the dramatic chemical structural differences between the two data sets (AID 372 and AID 565), and the models derived from the individual datasets may carry overwhelming localization features that might not be largely applicable to each other. This also leaves the gap to be filled in for a robust statistical model, better representation of physical chemical properties, enlarged and diversified dataset, and enhanced quality of the experimental accuracy in the future.

Nevertheless, this preliminary test using DT model as virtual screening technique yields encouraging enrichment for selection of active compounds when applied to another HTS activity dataset. It suggests that, despite of the very low similarities between the active compounds from the two HTS assays, certain common profiles of the active compounds can be extracted using the DT model, which can ultimately be very useful for virtual screening tasks.

\section{Conclusion}

In this study, we use derived DT models based on structural fingerprints of compounds to select biologically interesting compounds from HTS assay dataset. Four HTS assays were analyzed to determine to what extent the designed models can be applied to the compound libraries of an unknown domain. Our results suggest that the DT based models can be successfully used to derive common characteristics from HTS data, and the models can serve as filters to facilitate the selection of compounds against the same target. These DT models could also be used to eliminate HTS hits arising from data noise or those lacking statistical significance.

The development of the model is a learning process. Thus, the potential of the developed model is limited to the known active compounds and the properties used for training, and limited to the distribution of the compound collection to which the model is applied. With the growth in the number of compounds to be screened and the 
improvement over data quality produced with HTS assay, a more robust model could be developed with increased ability for selecting biologically interesting small molecules from a diverse compound library.

\section{Methods \\ Datasets}

There are over 500 assays deposited in the PubChem Bioassay database as of May 1st, 2007. About 200 of them have protein targets. In this study, four HTS assays were selected from the PubChem bioassay database. The criteria for the selection were that a substantial number of compounds have been tested in one assay and that the number of active compounds is in the hundreds to demonstrate statistical significance. These HTS assays are 5Hydroxytryptamine Receptor Subtype 1a (5HT1a) antagonists with PubChem BioAssay AID 612, HTS assay for screening 5HT1a agonists with AID 567, and PubChem bioassays AID 565, AID 372 for screening HIV-1 RTRNase $\mathrm{H}$ inhibitors. The number of structurally distinct compounds for these four bioassays and the number of active and inactive compounds are summarized in Table 1. Compound bioactivities outcomes have been described in a binary form, active and inactive, as specified by the assay depositor, and were retrieved from PubChem BioAssay database.

\section{PubChem Fingerprint System}

The numerical understanding of chemical structures is described by a binary substructure fingerprint generated by the PubChem Fingerprint System.

A substructure is a fragment of a chemical structure, such as a type of ring system, atom pairing, or atom environment (nearest neighbours). A fingerprint is an ordered list of binary $(1 / 0)$ bits. Each bit represents a Boolean determination of the presence of a fragment of a chemical structure. The PubChem fingerprint has a total of 881 bits and is composed of 7 sections such as Hierarchic Element Counts, chemical rings, and simple atom pairs, simple atom nearest neighbours, detailed atom information and two sections of SMARTS patterns. A detailed description and the full list of fingerprint bits can be accessed at ftp:// ftp.ncbi.nlm.nih.gov/pubchem/specifications/ pubchem fingerprints.txt.

The 2D structure of each compound was used to generate a binary substructure fingerprint.

\section{Decision Tree based Filtering Method}

DT is an acyclic graph in which its interior vertices specify testing of a single attribute of a feature vector and its leaves indicate the class of the decision [45-47]. It was constructed by recursively splitting the sample set, with each subset giving rise to one new vertex connected with an edge to its parent. This procedure continues until all samples at each leaf belong to the same class. The working flow of DT is similar to a logical tree structure that starts from the topmost node, and every decision of the node determines the direction of next node movement until the end of the tree branch node is reached. In this study, we performed DT analysis by utilizing the C4.5 core library [42].

As the filtering system is designed to choose the compounds of interest with certain features' commonalities to those compounds considered as active in the HTS assay, the binary representation of the activity outcomes were used to categorize these compounds. The structural fingerprint of every compound was processed by the PubChem fingerprints system subsequently for numerical description of the dataset for model training. To derive the models, the logical decision tree is then examined for error pruning, which is the removal of branches that are deemed to provide little or no gain in statistical accuracy of the model.

\section{Model Self-consistency evaluation}

The model self-consistency evaluation is performed using the 10 fold CV approach. As the HTS data are usually diversely distributed and not error free, the CV evaluation of the DT model is subjected to representatives from both the compounds used for training and for testing. Thus, for the balance between the computation cost and the evaluation of the model generalization ability, the 10 fold $\mathrm{CV}$ approach is chosen to assess the self-consistency of the model [48].

Under the assumption that the distribution of different subsets from one HTS assay is approximately equal, the quality of the model can be proven if the model built on the top of a portion of the data can be generalized to others during the self-consistency evaluation.

\section{Measurement of accuracies}

Model accuracy is measured by sensitivity, specificity, and a combined parameter called "overall accuracy." The sensitivity and specificity are defined as the following:

$$
\text { Sensitivity }=T P /(T P+F N)^{\prime} \quad \text { Specificity }=\mathrm{TN} /(\mathrm{TN}+\mathrm{FP})^{\prime}
$$

where the true positive (TP) is the number of compounds correctly predicted as active, false negative (FN) is the number of compounds incorrectly predicted as inactive, true negative (TN) is the number of compounds correctly predicted as inactive, and false positive (FP) is the number of compounds incorrectly predicted as active. Thus, the overall accuracy is defined as

$$
\text { Overall_Accuracy }=\frac{T P+T N}{T P+F N+T N+F P} \times 100 \% \text {. }
$$


In addition to compute sensitivity and specificity, to further evaluate the classification performance on a dataset containing imbalanced active and inactive compounds, Matthews correlation coefficient (MCC)[49] is also calculated as given by the following equation

$$
M C C=\frac{T P \cdot T N-F N \cdot F P}{\sqrt{(T P+F N)(T P+F P)(T N+F N)(T N+F P)}}
$$

MCC ranges from -1 to 1 , and suggests the randomness of the model.

\section{Use of DT models to select compounds of interest}

The best model is optimized by pruning the decision tree to minimize the classification errors. With the knowledge learned from the tested compounds and their bioactivities, one can apply these trained rules to filter new compounds that are likely to be active. In addition to the validation of the DT models with $\mathrm{CV}$ approach, application of models for the prediction of bioactivity classification have been attempted by using two HTS assays for identifying HIV-1 RT RNase inhibitors. Both of these two HTS assays are designed for the screening of the HIV-1 RT RNase $\mathrm{H}$ target. In spite of the differences in the design of HTS assays and in the selection of compound libraries, the underlying knowledge of those inhibition compounds was assumed to be similar or interpretable from one another. To this end, the optimized DT models of these two HTS assays were developed independently and were applied to examine the other compound collection as virtual experiments for identifying potential inhibitors.

\section{Computation Software}

The implementation of the Decision Tree-based models was based on PubChem Fingerprint System, OpenEye OEChem $\mathrm{C}++$ library, NCBI $\mathrm{C}++$ toolkit library, and the C4.5 core library.

\section{Authors' contributions}

All authors participated in development of the methods, discussions and preparation of the manuscript.

\section{Acknowledgements}

This research was supported by the Intramural Research Program of the $\mathrm{NIH}$, National Library of Medicine. We acknowledge the editorial assistance of the NIH Fellows Editorial Board.

\section{References}

I. Burbaum JJ, Sigal NH: New technologies for high-throughput screening. Curr Opin Chem Biol 1997, I(I):72-78.

2. Hann MM, Oprea TI: Pursuing the leadlikeness concept in pharmaceutical research. Curr Opin Chem Biol 2004, 8(3):255-263.

3. Cox B, Denyer JC, Binnie A, Donnelly MC, Evans B, Green DV, Lewis JA, Mander TH, Merritt AT, Valler MJ, et al: Application of highthroughput screening techniques to drug discovery. Prog Med Chem 2000, 37:83-133.

4. Walters WP, Namchuk M: Designing screens: how to make your hits a hit. Nat Rev Drug Discov 2003, 2(4):259-266.
5. Kevorkov D, Makarenkov V: Statistical analysis of systematic errors in high-throughput screening. J Biomol Screen 2005, I0(6):557-567.

6. Parker CN, Schreyer SK: Application of chemoinformatics to high-throughput screening: practical considerations. Methods Mol Biol 2004, 275:85-II0.

7. Lipinski CA: Drug-like properties and the causes of poor solubility and poor permeability. J Pharmacol Toxicol Methods 2000, 44(I):235-249.

8. Joseph-McCarthy D: Computational approaches to structurebased ligand design. Pharmacol Ther 1999, 84(2): 179-191.

9. Brown N, Zehender H, Azzaoui K, Schuffenhauer A, Mayr LM, Jacoby $E$ : A chemoinformatics analysis of hit lists obtained from high-throughput affinity-selection screening. J Biomol Screen 2006, II(2): I23-I30.

10. Tamura SY, Bacha PA, Gruver HS, Nutt RF: Data analysis of highthroughput screening results: application of multidomain clustering to the NCl anti-HIV data set. J Med Chem 2002, 45(I 4):3082-3093.

II. Martin YC, Kofron JL, Traphagen LM: Do structurally similar molecules have similar biological activity? J Med Chem 2002, 45(19):4350-4358.

12. Willett P, Barnard JM, Downs GM: Chemical Similarity Searching. J Chem Inf Comput Sci 1998, 38:983-996.

13. Rusinko A 3rd, Farmen MW, Lambert CG, Brown PL, Young SS: Analysis of a large structure/biological activity data set using recursive partitioning. J Chem Inf Comput Sci 1999, 39(6): $1017-1026$.

14. Xue L, Stahura FL, Bajorath J: Cell-based partitioning. Methods Mol Biol 2004, 275:279-290.

15. van Rhee AM, Stocker J, Printzenhoff D, Creech C, Wagoner PK, Spear KL: Retrospective analysis of an experimental highthroughput screening data set by recursive partitioning. J Med Chem 200I, 3(3):267-277.

16. van Rhee AM: Use of recursion forests in the sequential screening process: consensus selection by multiple recursion trees. J Chem Inf Comput Sci 2003, 43(3):94I-948.

17. Traeger M, Eberhart A, Geldner G, Morin AM, Putzke C, Wulf H, Eberhart LH: [Artificial neural networks. Theory and applications in anesthesia, intensive care and emergency medicine]. Anaesthesist 2003, 52(II): 1055-106I.

18. Cos P, Ying L, Calomme M, Hu JP, Cimanga K, Van Poel B, Pieters L, Vlietinck AJ, Berghe D Vanden: Structure-activity relationship and classification of flavonoids as inhibitors of xanthine oxidase and superoxide scavengers. J Nat Prod 1998, 6I(I):7I-76.

19. Kauffman GW, Jurs PC: QSAR and k-nearest neighbor classification analysis of selective cyclooxygenase- 2 inhibitors using topologically-based numerical descriptors. J Chem Inf Comput Sci 200 I, 4I(6): I 553-I560.

20. Mattioni BE, Jurs PC: Development of quantitative structureactivity relationship and classification models for a set of carbonic anhydrase inhibitors. J Chem Inf Comput Sci 2002, 42(I):94-102.

21. Pirard B, Pickett SD: Classification of kinase inhibitors using BCUT descriptors. J Chem Inf Comput Sci 2000, 40(6): I 43I-I 440.

22. Yan SF, Asatryan H, Li J, Zhou Y: Novel statistical approach for primary high-throughput screening hit selection. J Chem Inf Model 2005, 45(6): I 784-1790.

23. Hibert MF, Gittos MW, Middlemiss DN, Mir AK, Fozard JR: Graphics computer-aided receptor mapping as a predictive tool for drug design: development of potent, selective, and stereospecific ligands for the 5-HTIA receptor. J Med Chem 1988, $31(6): 1087-1093$

24. Loew GH, Villar HO, Jung W, Davies MF: Computer-aided drug design for the benzodiazepine receptor site. NIDA Res Monogr 1991, I | 2:43-61.

25. Marshall GR: Computer-aided drug design. Annu Rev Pharmacol Toxicol 1987, 27:193-213.

26. Ooms F: Molecular modeling and computer aided drug design. Examples of their applications in medicinal chemistry. Curr Med Chem 2000, 7(2): $141-158$.

27. Reddy MR, Erion MD: Computer-aided drug design strategies used in the discovery of fructose I, 6-bisphosphatase inhibitors. Curr Pharm Des 2005, I I (3):283-294.

28. Struthers RS, Rivier J, Hagler AT: Molecular dynamics and minimum energy conformations of GnRH and analogs. A meth- 
odology for computer-aided drug design. Ann $N$ Y Acad Sci 1985, 439:8I-96.

29. Vedani A: [Computer-Aided Drug Design: An Alternative to Animal Testing in the Pharmacological Screening]. Altex |991, 8(I):39-60.

30. Veselovsky AV, Ivanov AS: Strategy of computer-aided drug design. Curr Drug Targets Infect Disord 2003, 3(I):33-40.

3I. Jenwitheesuk E, Samudrala R: Prediction of HIV-I protease inhibitor resistance using a protein-inhibitor flexible docking approach. Antivir Ther 2005, 10(1):157-166.

32. Lybrand TP: Ligand-protein docking and rational drug design. Curr Opin Struct Biol 1995, 5(2):224-228.

33. Kokol P, Zorman M, Stiglic MM, Maleiae I: The limitations of decision trees and automatic learning in real world medical decision making. Medinfo 1998, 9(Pt I):529-533.

34. Aspinall P, Hill AR: Clinical inferences and decisions - II. Decision trees, receiver operator curves and subjective probability. Ophthalmic Physiol Opt 1984, 4(I):3I-38.

35. Sprogar M, Kokol P, Zorman M, Podgorelec V, Yamamoto R, Masuda $\mathrm{G}$, Sakamoto N: Supporting medical decisions with vector decision trees. Medinfo 200I, IO(Pt I):552-556.

36. Kuo WJ, Chang RF, Chen DR, Lee CC: Data mining with decision trees for diagnosis of breast tumor in medical ultrasonic images. Breast Cancer Res Treat 200I, 66(I):5I-57.

37. Wellman MP, Eckman MH, Fleming C, Marshall SL, Sonnenberg FA, Pauker SG: Automated critiquing of medical decision trees. Med Decis Making 1989, 9(4):272-284.

38. Decaestecker C, Remmelink M, Salmon I, Camby I, Goldschmidt D, Petein M, Van Ham P, Pasteels JL, Kiss R: Methodological aspects of using decision trees to characterise leiomyomatous tumors. Cytometry 1996, 24(I):83-92

39. Sabbagh A, Darlu P: Data-mining methods as useful tools for predicting individual drug response: application to CYP2D6 data. Hum Hered 2006, 62(3): I 19-134.

40. Kim HJ, Choo H, Cho YS, Koh HY, No KT, Pae AN: Classification of dopamine, serotonin, and dual antagonists by decision trees. Bioorg Med Chem 2006, I4(8):2763-2770.

4I. Ehrman TM, Barlow DJ, Hylands PJ: Virtual screening of Chinese herbs with random forest. J Chem Inf Model 2007, 47(2):264-278.

42. Quinlan JR: C4.5: programs for machine learning. San Mateo, Calif.: Morgan Kaufmann Publishers; 1993.

43. Baldi $\mathrm{P}$, Brunak S, Chauvin $\mathrm{Y}$, Andersen CA, Nielsen $\mathrm{H}$ : Assessing the accuracy of prediction algorithms for classification: an overview. Bioinformatics 2000, 16(5):412-424.

44. $\mathrm{Xu} \mathrm{H}$ : Retrospect and prospect of virtual screening in drug discovery. Curr Top Med Chem 2002, 2(I 2): | 305-1320.

45. Beerenwinkel N, Schmidt B, Walter H, Kaiser R, Lengauer T, Hoffmann D, Korn K, Selbig J: Diversity and complexity of HIV-I drug resistance: a bioinformatics approach to predicting phenotype from genotype. Proc Natl Acad Sci USA 2002, 99(I2):827I-8276.

46. Russell Stuart J, Norvig P: Artificial intelligence: a modern approach. Upper Saddle River, N.J.; [Great Britain]: Prentice Hall; 2003.

47. Quinlan JR: Induction of Decision Trees. Machine Learning 1986, I(I):8I-106.

48. Cyril G: Note on Free Lunches and Cross-Validation. Neural Computation 1997, 9(6): 1245-1249.

49. Matthews BW: Comparison of the predicted and observed secondary structure of T4 phage lysozyme. Biochim Biophys Acta 1975, 405(2):442-45।.
Publish with Biomed Central and every scientist can read your work free of charge

"BioMed Central will be the most significant development for disseminating the results of biomedical research in our lifetime. "

Sir Paul Nurse, Cancer Research UK

Your research papers will be:

- available free of charge to the entire biomedical community

- peer reviewed and published immediately upon acceptance

- cited in PubMed and archived on PubMed Central

- yours - you keep the copyright
BioMedcentral 